\title{
Article
}

\section{Flight Guardian: Autonomous Flight Safety Improvement by Monitoring Aircraft Cockpit Instruments}

Khan, Wasiq, Ansell, Darren, Kuru, Kaya and Muhammad, Bilal Available at https://clok.uclan.ac.uk/20869/

Khan, Wasiq orcid iconORCID: 0000-0002-7511-3873, Ansell, Darren orcid iconORCID: 0000-0003-2818-3315, Kuru, Kaya orcid iconORCID: 0000-00024279-4166 and Muhammad, Bilal (2018) Flight Guardian: Autonomous Flight Safety Improvement by Monitoring Aircraft Cockpit Instruments. Journal of Aerospace Information Systems, 15 (4). pp. 203-214.

It is advisable to refer to the publisher's version if you intend to cite from the work. http://dx.doi.org/10.2514/1.1010570

For more information about UCLan's research in this area go to http://www.uclan.ac.uk/researchgroups/ and search for <name of research Group>.

For information about Research generally at UCLan please go to http://www.uclan.ac.uk/research/

All outputs in CLoK are protected by Intellectual Property Rights law, including Copyright law. Copyright, IPR and Moral Rights for the works on this site are retained by the individual authors and/or other copyright owners. Terms and conditions for use of this material are defined in the policies page.

\section{CLoK}

Central Lancashire online Knowledge www.clok.uclan.ac.uk

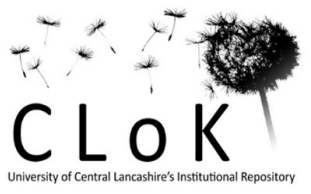




\title{
Flight Guardian: Autonomous Flight Safety Improvement by Monitoring Aircraft Cockpit Instruments
}

\author{
Wasiq Khan* \\ Manchester Metropolitan University, Manchester, England M15 6BH, United Kingdom \\ Darren Ansellı and Kaya Kuru \\ University of Central Lancashire, Preston, England PRI 2HE, United Kingdom \\ and \\ Muhammad Bilal 쪼 \\ University of California, Los Angeles, California 90095-7227 \\ DOI: $10.2514 / 1 . I 010570$
}

\begin{abstract}
During in-flight emergencies, a pilot's workload increases significantly, and it is often during this period of increased stress that human errors occur that consequently diminish the flight safety. Research studies indicate that many plane crashes can be attributed to ineffective cockpit instrument monitoring by the pilot. This paper entails the development of Flight Guardian ${ }^{\mathbb{I}}$ system being first of its kind that aims to provide efficient flight-deck awareness to improve flight safety while assisting the pilot in abnormal situations. The system is intended to be used in older aircraft that cannot easily or cost effectively be modified with modern digital avionic systems. One of the important features of the Flight Guardian system is being not physically connected to the aircraft, which avoids any impact on airworthiness or the need for recertification. For the first time, a composite of techniques including video analysis, knowledge representation, and machine belief representations are combined to build a novel flight-deck warning system. The prototype system is tested in both simulation-based laboratory and real flight environments under the guidance of expert pilots. The overall system performance is evaluated using statistical analysis of experimental results that proved the robustness of the proposed methodology in terms of automated warning generation in hazardous situations.
\end{abstract}

\section{Introduction}

$\mathbf{T}$ HE current generation of civil air transport aircraft are usually equipped with advanced intelligent warning systems (IWSs). One of the important aspects of these systems is the centralized alerting and monitoring system that displays information directly to the pilot. Typical aircraft warning systems monitor specific environmental properties that are difficult to observe or not observable by the pilot or flight crew. These systems generate warnings to alert the pilot about hazardous situations while keeping track of a number of parameters. When warnings occur in the cockpit, a pilot has procedures and actions to perform to mitigate the problem. During airborne emergencies, this requires a great deal of attention directed toward resolving the detected hazard that degrades the flight safety by increasing the pilot workload significantly. Research studies [1-4] address the consequences of multitasking on human brain and the ability to simultaneously deal with several parameters [5] as needed for cockpit monitoring by the human pilot.

The National Transportation Safety Board (NTSB) has long identified ineffective pilot monitoring of flight data to be problematic and therefore leading to crashes [6]. A safety study of crew-involved air carrier accidents between 1978 and 1990 [7] indicated that 31 out of 37 (i.e., 84\%) reviewed accidents were caused by ineffective monitoring of the cockpit instruments. A detailed study is presented by Iwadare and Oyama [] about recent plane crashes that constituted a huge number of fatal disasters in the aviation history. Seventy-five percent of these recent crashes were caused by pilot errors such as complacency, loss of control, lack of knowledge, distraction, lack of situational awareness, and lack of assertiveness [8]. These errors have marked some of the most fatal aviation disasters in history and have served as great learning examples on what to avoid in the future [9]. Likewise, statistics of aircraft crashes with respect to type of service, airlines, and number of fatalities per flight presented by Oster et al. [10] indicate a high number of accidents and fatalities caused by pilot error. Following the recent breakdown investigation reports, the NTSB made a number of recommendations to the Federal Aviation Administration forflight safety improvement [11] that can be achieved by facilitating the pilots with automated operational and safety-related information [12]. The NTSB also reported that the effect of technology and safety of general aviation operations can be improved by involving aircraft with a glass cockpit display [13]. The aforementioned statistics and reports manifest that a reliable automated monitoring of the cockpit instrument will improve the flight safety and would also decrease the pilot workload to recognize the nature of problem and eye scanning during emergencies.

In general, a cockpit warning system performs tasks of hazard detection, attention getting, display of resolution status and commands, and resolution guidance [14]. Current cockpit warning indicators give indications in hazardous situations but require a considerable amount of attention from the pilot. In addition, it takes significant amount of time to recognize the nature of problems and choose the right procedure [15]. To the best of our knowledge, there are no third-party systems in use to enable the external monitoring of cockpit instruments. The most commonly used existing warning systems are ground proximity warning system (GPWS) and traffic alert and collision avoidance system (TCAS), which are standalone applications that have improved aircraft safety [16]. Recent improvements in cockpit warning systems include enhanced GPWS, predictive wind-shear warning systems, and the anticipated future airborne separation assurance system. An agent-based cockpit safety system is

Received 19 May 2017; revision received 1 December 2017; accepted for publication 15 December 2017; published online XX epubMonth XXXX. Copyright $\odot$ 2018 by the American Institute of Aeronautics and Astronautics, Inc. All rights reserved. All requests for copying and permission to reprint should be submitted to CCC at www.copyright.com; employ the ISSN 2327-3097 (online) to initiate your request. See also AIAA Rights and Permissions www.aiaa.org/randp.

*Research Associate, School of Mathematics and Digital Technologies, JD Building; W.Khan@ mmu.ac.uk.

${ }^{\dagger}$ Aerospace Lead, School of Engineering, C\&T Building; DAnsell@UCLan.ac.uk.

Research Associate, School of Engineering, C\&T Building; KKuru@UCLan.ac.uk.

${ }^{\S}$ Assistant Researcher, Center for Environmental Implications of Nanotechnology; M.Bilal@UCLA.edu.

TFlight Guardian is the first generation of a disruptive cockpit technology funded by the National Aerospace Technology Exploitation Programme (grant NWC2-S006) to improve the safety of aircraft. Data available online at http://www.aerospace.co.uk/news/140915-natep-projects [retrieved ]. 
presented by Thatcher [17] by using three autonomous agents for aircraft path monitoring and cockpit behavior. The first agent is situated on the ground in the air traffic controller center of each airport. This agent uses an artificial neural network for training on a data set of successful landings at this airport. The data set consists of beliefs centered on the current landing, the aircraft's altitude, horizontal and lateral distance from the landing strip, speed, heading, type of aircraft, and weather conditions that affect the landing performance. In case of unusual events during the landing, a warning alarm is generated to the air traffic controller. The second and third agents are situated onboard the aircraft to monitor parameters such as aircraft position, heading, speed, and the pilot's behavior. These agents use harmonic topographic map and topographic products of experts for the prediction of hazardous situations.

Despite the technological advancements that have made IWS very sensitive to hazardous situations, tracing the exact problem and resolving it using a correct procedure is still a challenge that needs considerable attention [17]. Because of the increasing number of cockpit warning systems in modern aircraft, the need to prioritize warning messages to avoid nuisance alerts and provide a more intuitive human interface becomes more apparent [16]. As an example, one of the challenging factors in the development of an effective IWS is the potential for false alarms. Alarms could be erroneously triggered during real-time operation due to dynamics such as air turbulence, sunlight reflections, nighttime camera performance, and limitations of desired sensor/camera technology that could misidentify objects in the cockpit. These false alarms can result in a pilot's general distrust of the system. The problems associated with false and nuisance alarms were demonstrated in the design of the first generation of TCAS system for commercial aircraft. These systems had such a high nuisance alarm rate that pilots failed to trust the system's validity as an alerting device. This situation could be analogous to what may happen in the automotive crash-warning domain [18].

The aforementioned studies indicate the significance of a reliable, intelligent, easy to expand, and supportive warning system for pilot assistance. The Flight Guardian (FG) prototype presented in this paper provides such a platform using composite of techniques to improve the flight safety and pilot support. Image processing techniques are employed for automated dial reading (ADR) to produce instantaneous state information of the cockpit instruments. For the first time, domain knowledge with respect to the appropriate range of cockpit instrument readings is presented, which is gathered from domain experts to prove the proposed concept of FG system. The theory of evidence is used to build the decision support system (DSS) that combines beliefs from two cockpit instruments for intelligent reasoning. In abnormal conditions, the FG system generates spoken and visual alerts as well as resolution advice after diagnosing the problem, which helps the pilot to choose the corresponding procedure for mitigating the hazardous situations.

\section{Materials and Methods}

A composite of techniques was sequentially combined to build an FG system as shown in Fig. 1 . The inputs to the system consist of two video streams of cockpit instruments (analog) acquired by the camera devices, which were then processed by a sequence of ADR, data representation, transformation, and probabilistic modeling approaches to inform a final decision-making component to decide if flight parameters need to be adjusted to return the aircraft to a safe state.

\section{A. Data Acquisition and Automated Dial Reading}

In the first step, two fixed-position camera devices are placed in front of the airspeed (ASp) and revolutions per minute (RPM) instruments to acquire the corresponding video streams as shown in Fig. 1. Microsoft Lifecam Cinema devices are used that produce 30 frames per second. The video streams are then processed to generate instantaneous snapshots (i.e., images) with a frequency of $0.5 \mathrm{~Hz}$ and forwarded to the ADR component. The primary objective of the ADR is to provide instantaneous state evidences (i.e., current readings) for the cockpit instruments to the DSS. With regard to ADR, different approaches are explored that have been used in the literature. For instance, Feng and Zhao [19] presented a background-subtraction-based approach that subtracts the current image frame from a reference frame to identify the target object's movements in the scene. Similarly, pattern matching [20] provides the object segmentation based on the features extracted from the input image. Likewise, Gellaboina et al. [21] presented a polar representation of the dial gauge image to identify the needle. These approaches are generic and simple to implement but exhibit poor performance in the presence of specular highlights [21] that may occur due to sunlight reflections on the cockpit instruments during flight.

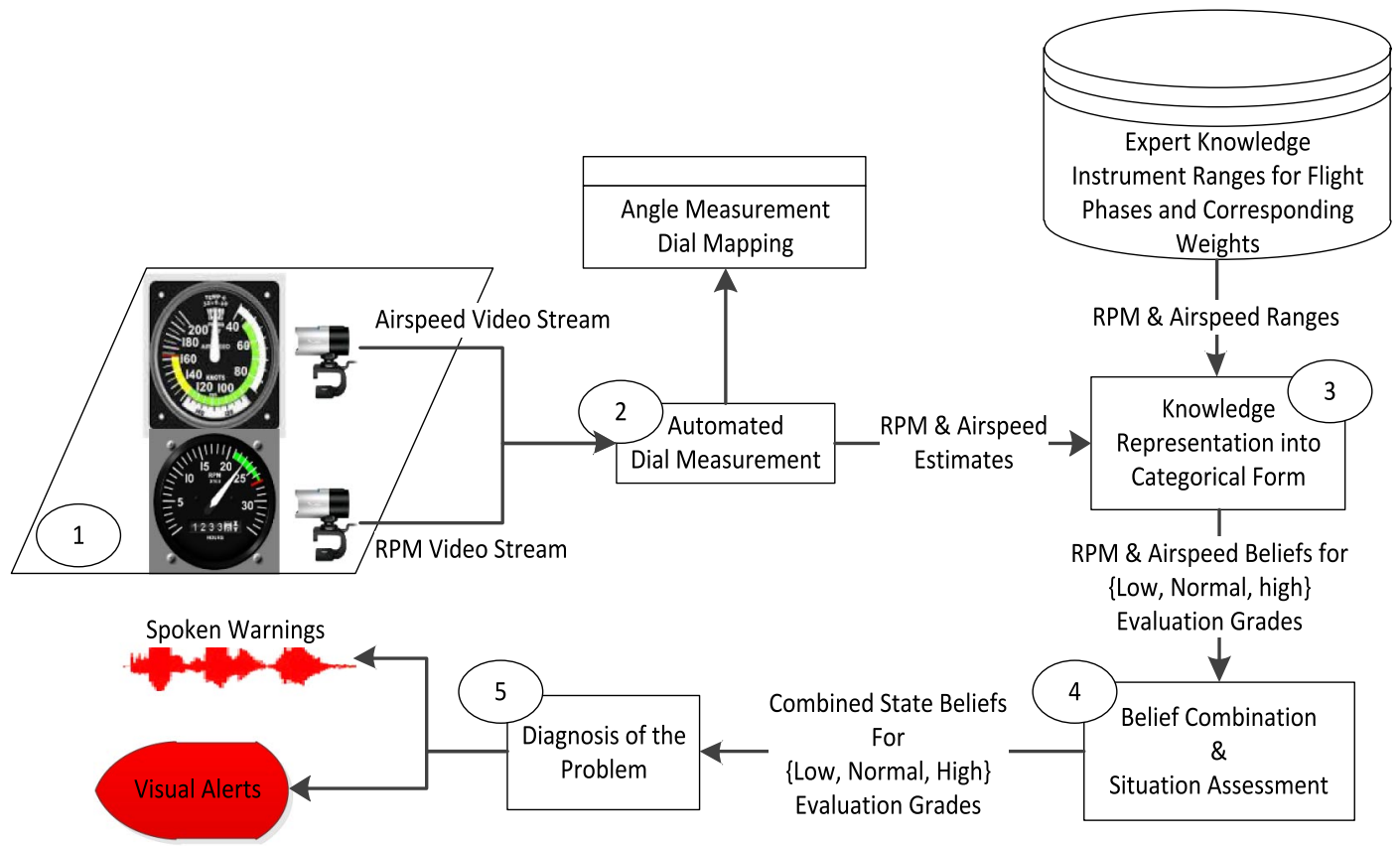

Fig. 1 Sequential processing of the deployed methods for the flight guardian system composition. 


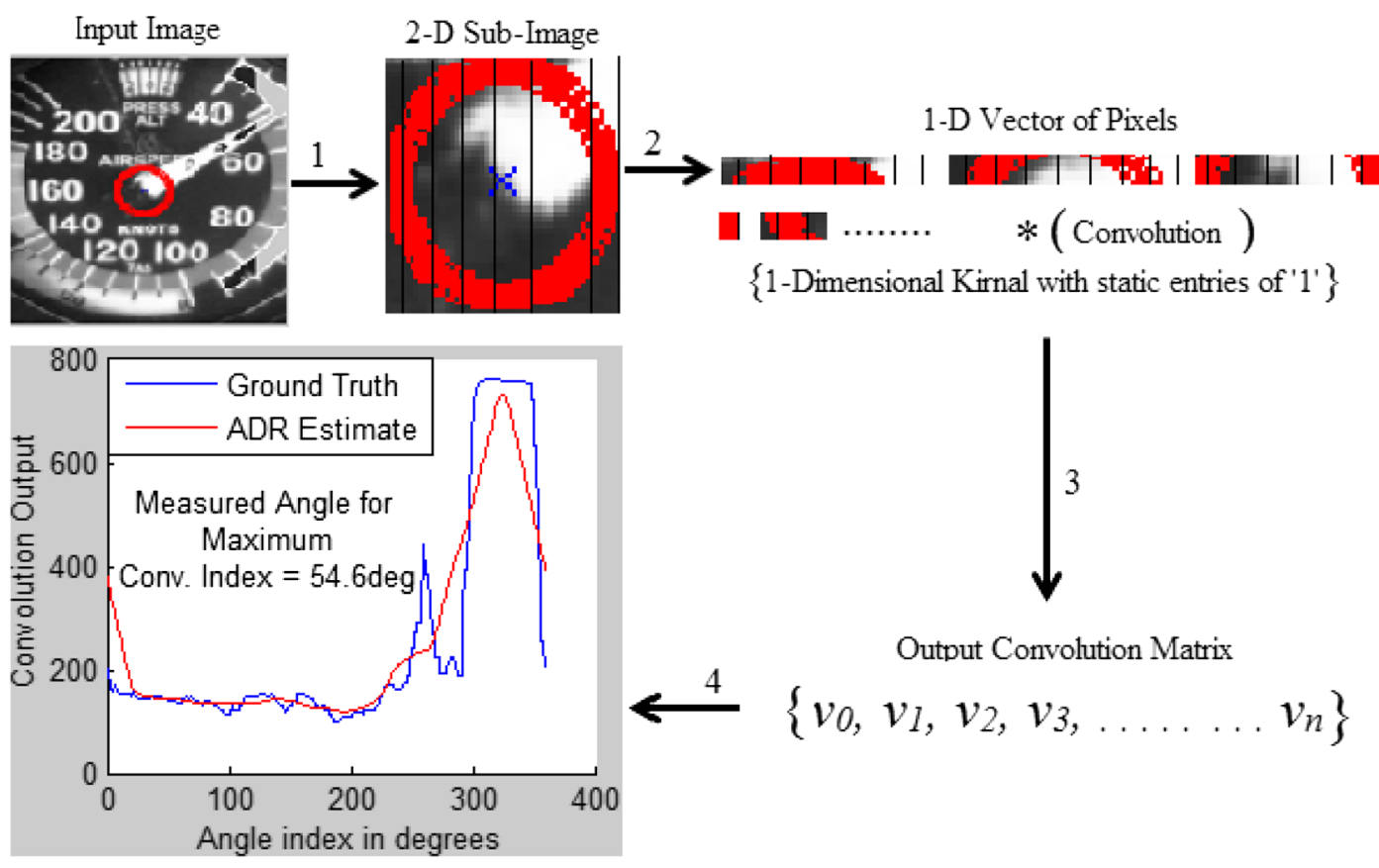

Fig. 2 Estimation of the gauge angular position within the airspeed dial using convolution.

To deal with this issue, a convolution-based ADR approach is used to detect the needle within the ASp and RPM dials, as presented in our previous work [22]. Because of the circular shape and needle locations in both dials, the focus of the camera devices is fixed at the center coordinates of the cockpit instruments as shown in Fig. 2. It can be observed that all pixels are black within a limited circular range (red circle in Fig. 2) around the image center except the white pixels occupied by the needle. Therefore, only a subimage (within the red circle) is needed to identify the needle position in the cockpit instruments. In the second step, a convolution function with a static one-dimensional kernel is used to identify the needle and hence its position in the dial. Because the entire pixels occupied by the target needle are white, the entries of the kernel vector are set to 1 such that it produces maximum convolution output for only white pixels within the cropped subimage. The entire convolution process between selected subimage pixels $x$ and a kernel $h$ over a predefined interval is described as the following pseudocode.

Inputs:

One-dimensional vector ' $x$ ' for target subimage pixels

One-dimensional kernel vector $h=\{1,1,1, \ldots$, length $(x)\}$

Output:

Angle $\theta$; maximum convolution index in output vector $v$

for all indexes $i$ in $x$, do

$$
v[i]=0 \text {; }
$$

for all indexes $j$ in $h$ do

$$
v[i]=v[i]+x[i-j]^{*} h[j]
$$

end for

end for

max index $=$ index of maximum value in the output vector $v$

$$
\theta=360 /(\text { length }- \text { of }-x)^{*} \max \text { index }
$$

\section{B. Knowledge Representation}

In the next step, expert knowledge is acquired from domain experts at the Great Circle** and related information booklets [23,24] that demonstrate the ideal ranges for cockpit instruments (i.e., airspeed and engine revolutions per minute) of Cessna C172SP aircraft for different flight phases as presented in Table 1 . Based on the selected model for evidence combination and situation assessment, the numeric data in the form of cockpit instruments ranges and $\bar{A} D R$ measurements are transformed into the categorical form (i.e., low, normal, high). The Gaussian, $Z$, and $S$ membership functions (MFs) are used to interpret the numeric data into a categorical form with a degree of belief. Table 1 presents detailed information about the instrument ranges and the parameter values for these MFs with respect to different flight phases. These parameters describe the MFs in terms of their ranges within the data.

The parameters $a$ and $b$ locate the lower and upper bounds within the input numerical data respectively, for $Z$ and $S$ MFs. Similarly, $c$ and $\sigma$ represent the center and width parameters, respectively, for the Gaussian MF. A detailed discussion about these member functions and their representation is presented in [25]. These functions take the ASp and RPM reading estimates along with the expert knowledge and transform the

**The Great Circle Ltd. and the University of Central Lancashire are working in partnership on the Flight Guardian Project. Data available online at http://www. thegreatcircle.co.uk [retrieved 
Table 1 Cockpit instruments ranges for different flight phases and parameter setting for Gaussian, $Z$, and $S$ member functions

\begin{tabular}{|c|c|c|c|c|c|c|c|c|c|c|c|c|c|c|}
\hline \multirow[b]{3}{*}{ Flight phases } & \multirow[b]{3}{*}{ Engine noise ranges } & \multirow[b]{3}{*}{ Airspeed ranges, kt } & \multicolumn{6}{|c|}{$\begin{array}{l}\text { MF parameters for airspeed } \\
\text { representation }\end{array}$} & \multicolumn{6}{|c|}{ MF parameters for RPM representation } \\
\hline & & & \multicolumn{2}{|c|}{$Z$} & \multicolumn{2}{|c|}{ Gaussian } & \multicolumn{2}{|c|}{$S$} & \multicolumn{2}{|c|}{$Z$} & \multicolumn{2}{|c|}{ Gaussian } & \multicolumn{2}{|c|}{$S$} \\
\hline & & & $a$ & $\bar{b}$ & $c$ & $\sigma$ & $a$ & $b$ & $a$ & $b$ & $c$ & $\sigma$ & $a$ & $B$ \\
\hline Takeoff & $\begin{array}{c}\text { Low: } \mathrm{RPM}<2000 \\
\text { normal: } 2000<\mathrm{RPM}<2600 \\
\text { high: } \mathrm{RPM}>2600\end{array}$ & $\begin{array}{c}\text { Low: Asp }<5 \\
\text { normal: } 5<\mathrm{ASp}<60 \text {; } \\
\text { high: Asp }>65\end{array}$ & 0 & 3 & 30 & 30 & 60 & 70 & 1800 & 2000 & 2200 & 400 & 2400 & $\overline{2800}$ \\
\hline Climb & $\begin{array}{c}\text { Low: } \mathrm{RPM}<2000 \text {; } \\
\text { normal: } 2000<\mathrm{RPM}<2600 \text {; } \\
\text { high: } \mathrm{RPM}>2600\end{array}$ & $\begin{array}{l}\text { Low: Asp }<55 \\
\text { normal: } 60<\text { Asp }<80 \\
\text { high: Asp }>90\end{array}$ & 45 & 60 & 72 & 20 & 75 & 95 & 1800 & 2000 & 2200 & 400 & 2400 & 2800 \\
\hline Cruise & $\begin{array}{c}\text { Low: } \mathrm{RPM}<1750 \\
\text { normal: } 1800<\mathrm{RPM}<2500 \text {; } \\
\text { high: } \mathrm{RPM}>2600\end{array}$ & $\begin{array}{c}\text { Low: Asp }<60 ; \\
\text { normal: } 70<\text { Asp }<100 ; \\
\text { high: Asp }>110\end{array}$ & 50 & 85 & 85 & 25 & 85 & 115 & 1700 & 1900 & 2150 & 450 & 2350 & 2700 \\
\hline Cruise & $\begin{array}{c}\text { Low: } \mathrm{RPM}<1600 \\
\text { normal: } 1800<\mathrm{RPM}<2000 \\
\text { high: } \mathrm{RPM}>2300\end{array}$ & $\begin{array}{c}\text { Low: Asp }<60 \\
\text { normal: } 70<\text { Asp }<90 \\
\text { high: Asp }>100\end{array}$ & 50 & 70 & 80 & 20 & 75 & 115 & 1500 & 1900 & 2000 & 350 & 2150 & 2350 \\
\hline
\end{tabular}

data into the degree of beliefs for multiple evaluation grades corresponding to each flight phase. These degrees of beliefs are then forwarded to an evidence combination stage for the flight situation assessment. Figure 3 demonstrates the categorical form of data representation for the ASp ranges in a cruise flight phase presented in Table $\underline{1}$ using the aforementioned member functions and the corresponding parameters.

\section{Evidence Combination}

The categorical state information from the previous step is then forwarded to the evidence combination process that uses the Dempster-Shafer (DS) theory of evidence also considered as a generalization to the Bayesian theory in such a way that it can handle the degree of ignorance [26]. The DS provides the best state estimate by combination of evidences from multiple information resources while taking into account the corresponding weights. One of the interesting advantages of the DS is the model simplicity for the complex multilayered situations where the system can be decomposed into many layers of simpler states and then the beliefs can be propagated upward, combined with sibling layer states to get overall belief [27]. A detailed study on DS advantages, disadvantages, and its application areas is presented in [26,27]. In the proposed study, the DS performs the flight situation assessment task using beliefs from two cockpit instruments (i.e., ASp and RPM) in different flight phases. Mathematical formulation of the DS for the FG system is presented in the following steps.

Let $E=\{\mathrm{ASp}, \mathrm{RPM}\}$ represent the set of basic attributes representing the evidences produced by ASp and engine revolution speed, respectively. The relative weights for the basic attributes are equally distributed according to the pilot instructions such that $0 \leq \omega_{i} \leq 1$ and the weights sum to 1 . The distinctive evaluation grades for each attribute are defined as a set of three entities, i.e.,

$$
H=\{\text { low, normal, high }\}
$$

For each attribute in $E$ and evaluation grade $H$, a degree of belief $\beta_{n}$ is assigned by the member functions described earlier, which denotes the source's level of confidence when assessing the level of fulfillment of a certain property.

\section{Basic Probability Assignments for Each Basic Attribute}

Let $m_{n, i}$ be a basic probability mass representing the degree to which the $i$ th basic attribute is assessed. A hypothesis that the general attribute is assessed to the $n$th evaluation grade $H_{n}$ can be presented as

$$
m_{n, i}=\omega_{i} \beta_{n, i}
$$

where $n=3$ is the number of evaluation grades (i.e., low, normal, high). The remaining probability mass $m_{H, i}$ unassigned to each basic attribute is calculated as

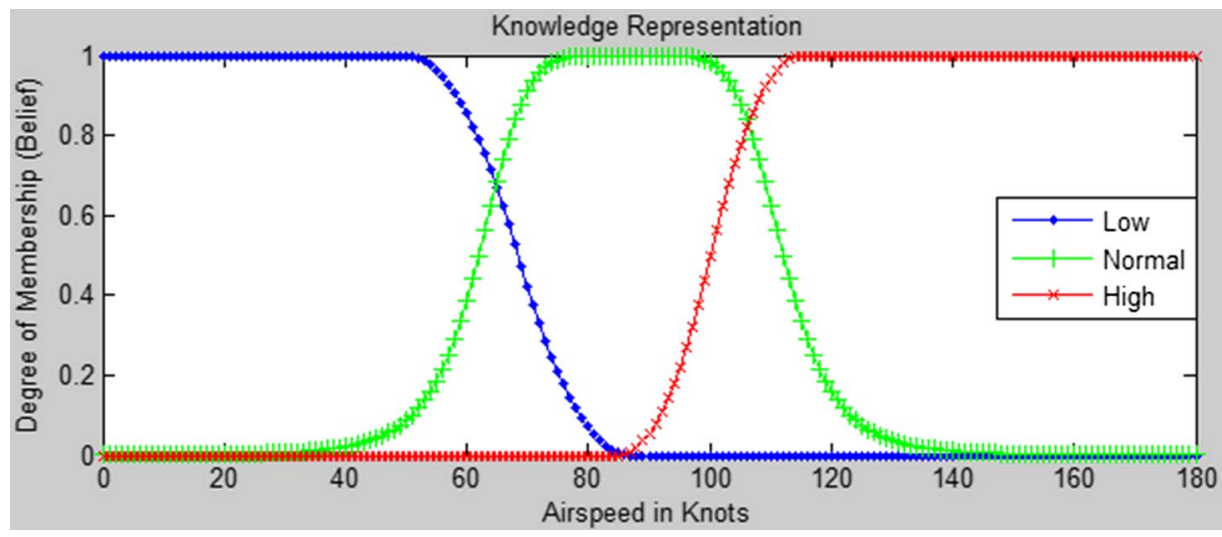

Fig. 3 Categorical representation for the airspeed ranges in cruise phase using Gaussian, $S$, and $Z$ membership functions. 


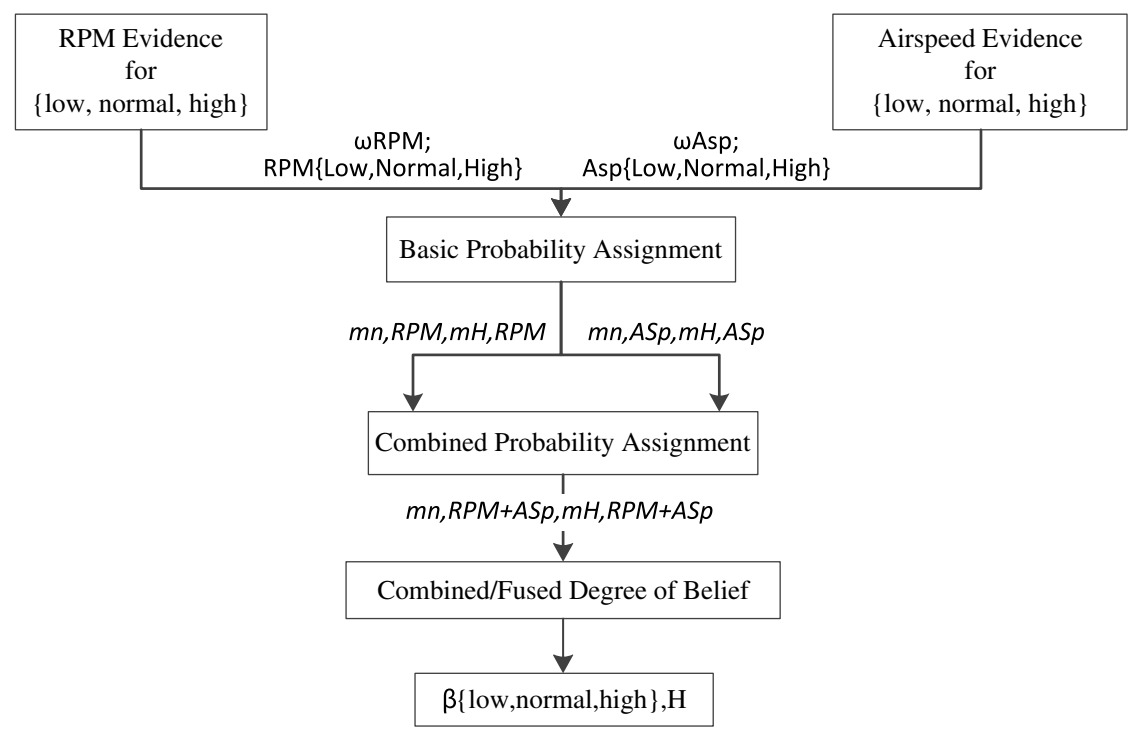

Fig. 4 Combining the beliefs from multiple cockpit instruments for being in low, medium, and high states.

$$
m_{H, i}=1-\sum_{n=1}^{N} m_{n, i}=1-\omega_{i} \sum_{n=1}^{3} \beta_{n, i}
$$

The remaining probability mass is further decomposed into $\bar{m}_{H, i}$ and $\tilde{m}_{H, i}$ as

$$
\begin{gathered}
\bar{m}_{H, i}=1-\omega_{i} \\
\tilde{m}_{H, i}=\omega_{i}\left(1-\sum_{n=1}^{3} \beta_{n, i}\right)
\end{gathered}
$$

Equation (4) measures the degree to which final attributes have not yet been assessed to individual grades due to the relative importance of basic attributes after their aggregation. Equation (5) measures the degree to which final attributes cannot be assessed to individual grades due to the incomplete assessments for basic attributes.

2. Combined Probability Assignments

In next step, the probability masses of the basic attributes are aggregated to form a single assessment for the low/normal/high grades. The combined probability masses can be generated using the following set of recursive evidence reasoning equations:

$$
\begin{aligned}
& \left\{H_{n}\right\}: \\
& m_{n, i+1}=K_{i+1}\left[m_{n, i} \cdot m_{n, i+1}+m_{H, i} \cdot m_{n, i+1}+m_{n, i} \cdot m_{H, i+1}\right] \\
& n=1, \ldots, N
\end{aligned}
$$

where $i=\{1,2\}$ represents the number of basic attributes. In the preceding equation, $m_{n, 1} \cdot m_{n, 2}$ measures the degree of both attributes \{ASp, RPM\} supporting the general attribute of decision to be assessed to $H_{n}$. The term $m_{n, 1} \cdot m_{H, 2}$ measures the degree of only the first attribute \{ASp\} supporting decision to be assessed to $H_{n}$. The term $m_{H, 1} \cdot m_{n, 2}$ measures the degree of only the second attribute \{RPM\} supporting final belief to be assessed to $H_{n}$ :

$$
\begin{gathered}
m_{H, i}=\bar{m}_{H, i}+\tilde{m}_{H, i} \\
\tilde{m}_{H, i+1}=K_{i+1}\left[\tilde{m}_{H, i} \cdot \tilde{m}_{H, i+1}+\bar{m}_{H, i} \cdot \tilde{m}_{H, i+1}+\bar{m}_{H, i+1} \cdot \tilde{m}_{H, i}\right] \\
\bar{m}_{H, i+1}=K_{i+1}\left[\bar{m}_{H, i} \cdot \bar{m}_{H, i+1}\right] \\
K_{i+1}=\left[1-\sum_{t=1}^{N=3} \sum_{\substack{j=1 \\
j \neq t}}^{N=3} m_{t, i} \cdot m_{j, i+1}\right]^{-1}
\end{gathered}
$$


In Eq. ( $\underline{8}), \tilde{m}_{H, 1} \cdot \tilde{m}_{H, 2}$ measures the degree to which the final attribute cannot be assessed to any individual grades low, normal, and high due to the incomplete assessments for both attributes $\{\mathrm{ASp}, \mathrm{RPM}\}$. The term $\bar{m}_{H, 1} \cdot \tilde{m}_{H, 2}$ measures the degree to which final attributes cannot be assessed due to the incomplete assessments for $\{\mathrm{ASp}\}$ only. In Eq. (9), $\bar{m}_{H, 1} \cdot \bar{m}_{H, 2}$ measures the degree to which final attributes have not been assessed yet to individual grades due to the relative importance of $\{\mathrm{ASp}\}$ and $\{\mathrm{RPM}\}$ after $\{\mathrm{ASp}\}$ and $\{\mathrm{RPM}\}$ have been aggregated. The normalization factor $K$ is used to normalize $m_{n}, m_{H}$ such that

$$
\sum_{n=1}^{2} m_{n}+m_{H}=1
$$

\section{Calculation of the Combined Degrees of Belief}

Finally, let $c \beta_{n}$ denote the combined degree of belief that the situation is assessed to the grade $H_{n}$, which is generated by combining the assessments for all the associated basic attributes $E=\{\mathrm{ASp}, \mathrm{RPM}\}$; then, $c \beta_{n}$ is calculated by

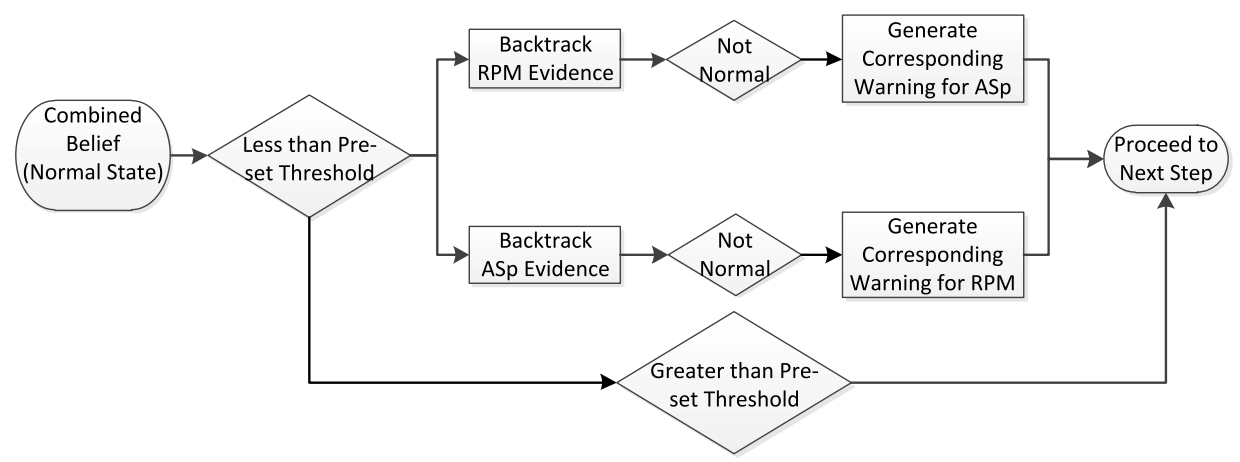

Fig. 5 Diagnosis of hazard cause by backtracking the individual beliefs from RPM and airspeed, corresponding ADR measurements, and domain knowledge in case of abnormal situation.

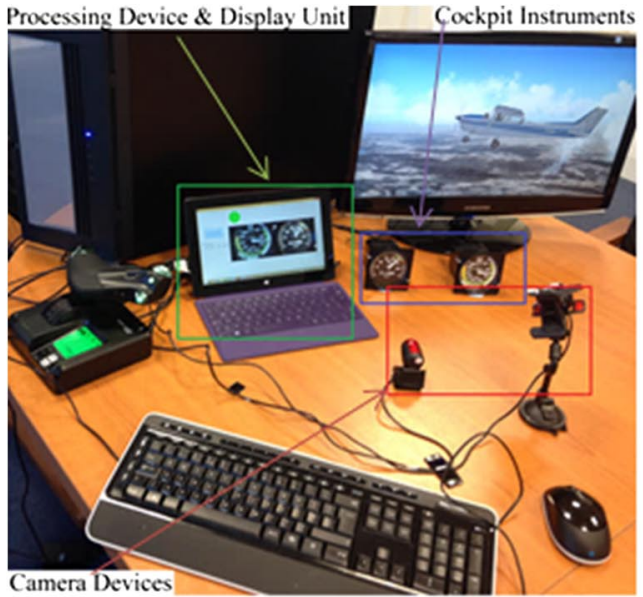

a) Lab environment

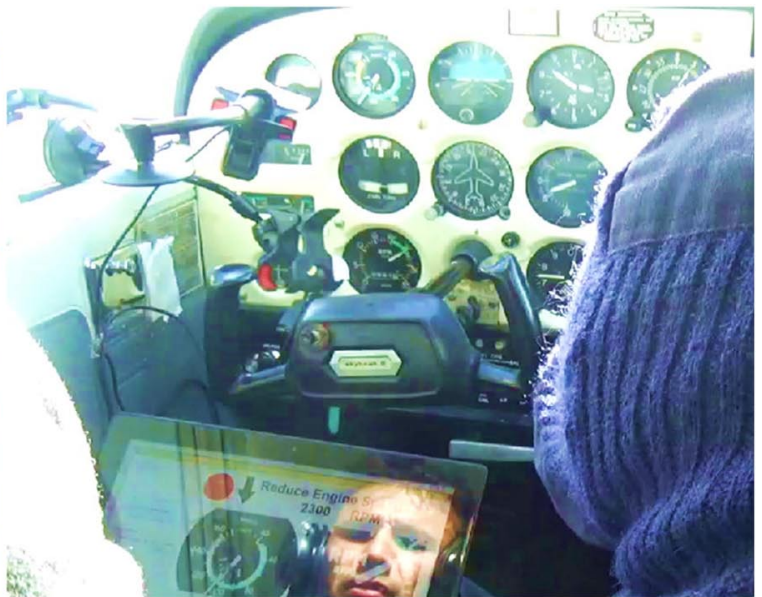

b) Real flight environment

Fig. 6 Experimental setup for the flight guardian system in simulated and real flight environments.

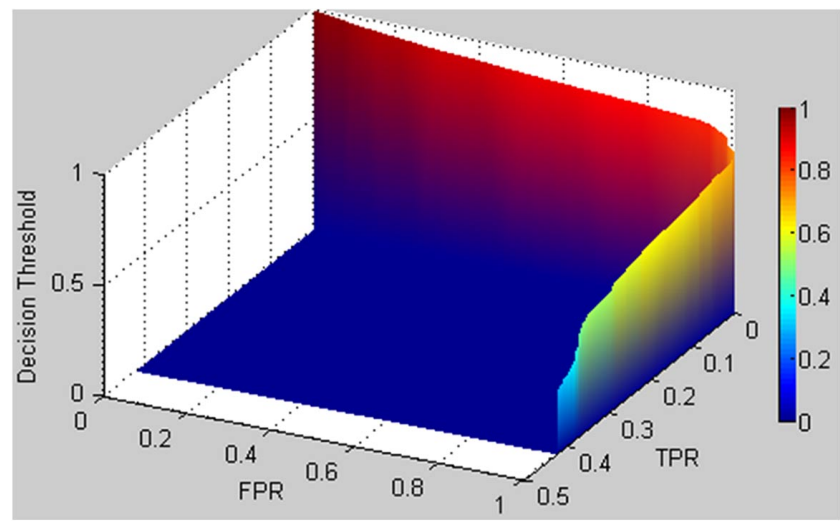

Fig. 7 ROC curve for the optimal decision boundary selection using true hazard detection and false alarms rate. 
Table 2 FG performance for different automated dial reading approaches in a real and simulated flight environments

\begin{tabular}{|c|c|c|c|c|c|c|c|c|c|c|}
\hline \multirow[b]{2}{*}{ ADR approach } & \multicolumn{5}{|c|}{ Instances/scenarios } & \multirow[b]{2}{*}{$\begin{array}{c}\text { True positive } \\
\text { rate, } \%\end{array}$} & \multirow[b]{2}{*}{$\begin{array}{c}\text { True negative } \\
\text { rate, } \%\end{array}$} & \multirow[b]{2}{*}{ Accuracy, $\%$} & \multirow[b]{2}{*}{$\begin{array}{c}\text { Execution } \\
\text { time, } \mathrm{S}\end{array}$} & \multirow[b]{2}{*}{ Frequency, $\mathrm{H}$} \\
\hline & Total & $\mathrm{TP}$ & $\mathrm{TN}$ & FP & $\mathrm{FN}$ & & & & & \\
\hline \multicolumn{11}{|c|}{ Live flight test for takeoff, climb, cruise, and descent phases } \\
\hline Convolution & 1350 & 450 & 863 & 37 & 0 & 100 & 95.9 & 97.20 & 0.52 & 0.5 \\
\hline Background subtraction & 1350 & 200 & 600 & 300 & 250 & 44.40 & 66.66 & 59.25 & 0.41 & 0.5 \\
\hline Pattern matching & 675 & 160 & 355 & 95 & 65 & 71.11 & 78.80 & 76.29 & 2.12 & 0.25 \\
\hline \multicolumn{11}{|c|}{ Lab test for takeoff, climb, cruise, and descent phases } \\
\hline Convolution & 1805 & 400 & 1405 & 0 & 0 & 100 & 100 & 100 & 0.52 & 0.5 \\
\hline Background Subtraction & 1805 & 300 & 1004 & 401 & 100 & 75.00 & 71.42 & 72.02 & 0.41 & 0.5 \\
\hline Pattern matching & 902 & 177 & 650 & 52 & 23 & 88.75 & 92.52 & 91.68 & 2.12 & 0.25 \\
\hline
\end{tabular}

$$
\begin{gathered}
\left\{H_{n}\right\}: c \beta_{n}=\frac{m_{n, i}}{1-\bar{m}_{H, i}} \quad n=1, \ldots, N \\
\{H\}: c \beta_{H}=\frac{\tilde{m}_{H, i}}{1-\bar{m}_{H, i}}
\end{gathered}
$$

Equation (12) for $c \beta_{H}$ measures the belief that is left unassigned during the assessments. The set of Eqs. (1-12) provides the combined degree of beliefs for the low, normal, and high grades that are further used for the decision making about a normal or abnormal flight situation.

A flight state is considered as "abnormal" if the combined belief for "normal" state is less than a predefined decision threshold as shown in Fig. 5. In the case of an abnormal situation, the problem is identified by automated analysis of the corresponding instrument state information (i.e., backtracking individual beliefs) and corresponding measurements retrieved from the ADR to identify the required adjustments with regard to the domain knowledge presented in Table 1. Finally, a warning message is generated to the pilot that provides precise information about the diagnosed problem and instructions to return the aircraft to a safe state.

\section{Experimental Setup and System Evaluation}

Performance of the proposed system is evaluated using different experimental setup that includes the following.

\section{Laboratory Environment Using a Flight Simulator}

In a laboratory environment, experiments are conducted in a simulated flight using the Flight Simulator X (FSX) platform. Two fixed-position camera devices are placed directly in front of the ASp and RPM instruments that export continuous video streams to the processing device. The video streams are processed and converted into instantaneous snapshots (i.e., images) with a frequency of $0.5 \mathrm{~Hz}$ and forwarded for further processing. Microsoft Lifecam Cinema devices are used, which produce $30 \mathrm{fps}$. In addition, a virtual software camera (i.e., manyCam) is used to provide the zoom function to physical camera devices. The domain knowledge along with the simulated flight data are used to know the ground truth (i.e., true state) of whether the system was in a normal or abnormal situation, which is then compared to the DSS output for that particular instance to evaluate the performance. Because the output warnings generated by the FG system are in the binary form of normal and abnormal situations, the statistical metrics for a binary classifier are used for the DSS performance evaluation that includes 1) sensitivity, 2) specificity, and 3) accuracy.

The sensitivity indicates the probability that an instance is classified as a warning with high confidence in the case of an actual hazard. Specificity shows the probability that an instance is classified as a normal situation with high confidence when it is indeed a normal situation and therefore no warning is generated by the DSS. Accuracy is a percentage of all the instances that were correctly classified. Detailed experiments are conducted for various conditions in terms of different flight phases (i.e., takeoff, climb, cruise, and descent), decision boundaries, varying light intensity, and environmental dynamics to evaluate the ADR performance. Figure 6 a shows the experimental setup in the lab environment for a simulated flight.

\section{Real Aircraft Flight Environment Under the Guidance of Experienced Pilots}

Despite the high expenses associated with the real flight experimental setup, the proposed FG prototype is tested twice using Cessna C172SP aircraft in the presence of two expert pilots (one pilot in each flight). The selected method is evolved according to the test outcomes from each flight test to mitigate the real flight challenges. There is no significant change in the experimental setup for the real flight environment, except that the

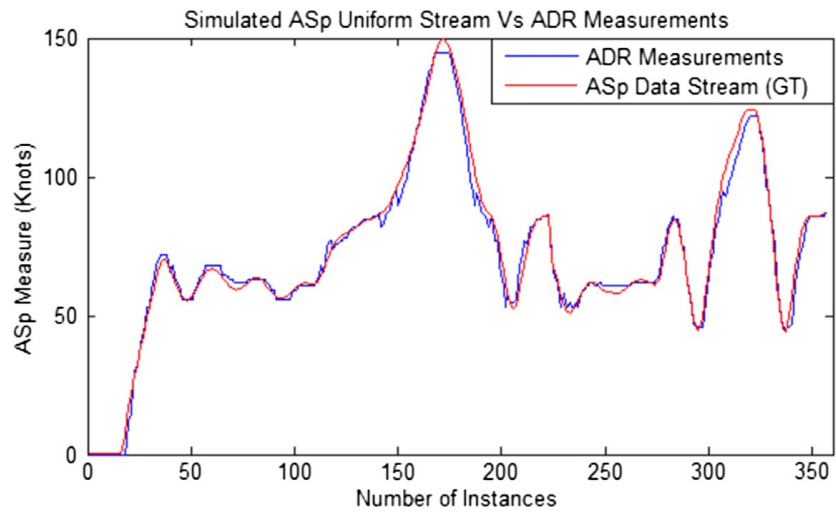

Fig. 8 Comparison between simulated uniform data stream vs ADR measurements. 
Table 3 Performance evaluation with varying lighting conditions using different automated dial reading approaches in the simulated flight environment

\begin{tabular}{|c|c|c|c|c|c|c|c|c|c|c|c|}
\hline \multirow[b]{2}{*}{$\underline{\text { Lighting conditions }}$} & \multirow[b]{2}{*}{$\alpha$} & \multirow[b]{2}{*}{$\beta$} & \multicolumn{3}{|c|}{ Convolution-based ADR } & \multicolumn{3}{|c|}{ Pattern-matching-based ADR } & \multicolumn{3}{|c|}{ Background-subtraction-based ADR } \\
\hline & & & $\begin{array}{c}\text { False } \\
\text { alarm, \% }\end{array}$ & $p$-value & RMSE & $\begin{array}{c}\text { False } \\
\text { alarm, \% }\end{array}$ & $p$-value & RMSE & $\begin{array}{c}\text { False } \\
\text { alarm, \% }\end{array}$ & $p$-value & RMSE \\
\hline Room light & 0 & 1 & 0 & 0.01 & 0.25 & 5.80 & 0.09 & 4.50 & 22.40 & 0.29 & 13.84 \\
\hline Lower ambient light & 0 & 0.5 & 0 & 0.01 & 0.31 & 6.89 & 0.09 & 5.81 & 27.01 & 0.31 & 15.32 \\
\hline Dark ambient light & 0 & 0.3 & 0 & 0.01 & 0.35 & 8.09 & 0.11 & 5.95 & 29.20 & 0.33 & 17.44 \\
\hline Higher ambient light & 0.5 & 1 & 0 & 0.01 & 0.14 & 7.91 & 0.10 & 5.71 & 27.33 & 0.32 & 14.88 \\
\hline Bright ambient light & 0.7 & 1 & 0 & 0.01 & 0.22 & 8.87 & 0.12 & 6.23 & 28.15 & 0.34 & 16.01 \\
\hline Direct specular light & -- & -- & 1.7 & 0.02 & 0.64 & 17.03 & 0.19 & 8.13 & 42.00 & 0.49 & 31.15 \\
\hline Indirect specular light & -- & -- & 0 & 0.01 & 0.17 & 6.23 & 0.09 & 4.81 & 34.92 & 0.43 & 24.92 \\
\hline
\end{tabular}

camera position is placed such that it does not interfere with the pilot's internal or external view, as shown in Fig. $6 \mathrm{~b}$. However, the automated ground truth data extraction from the cockpit instruments in a real flight environment is not possible, specifically in older aircraft. In this scene, video data are recorded while testing the FG system in real flight by creating different hazardous scenarios by the pilot. The recorded video is then converted into image instances with a frequency of $0.5 \mathrm{~Hz}$ (i.e., execution time), and the ground truth (i.e., true states) is generated manually to retrieve the statistical results using the aforementioned binary classification metrics. In addition, the overall system performance is evaluated for multiple ADR approaches to identify the best one in terms of accuracy. Finally, a combined statement is given by the pilots at the end of flight tests, which empowers the significance of the proposed system for the flight safety and pilot assistance.

\section{Results and Discussions}

The experimental results are achieved to evaluate the FG system performance under the experimental setup described earlier. These statistical results are highly dependent upon the weight assigned to ASp and RPM measurements. However, both instruments are weighted equally
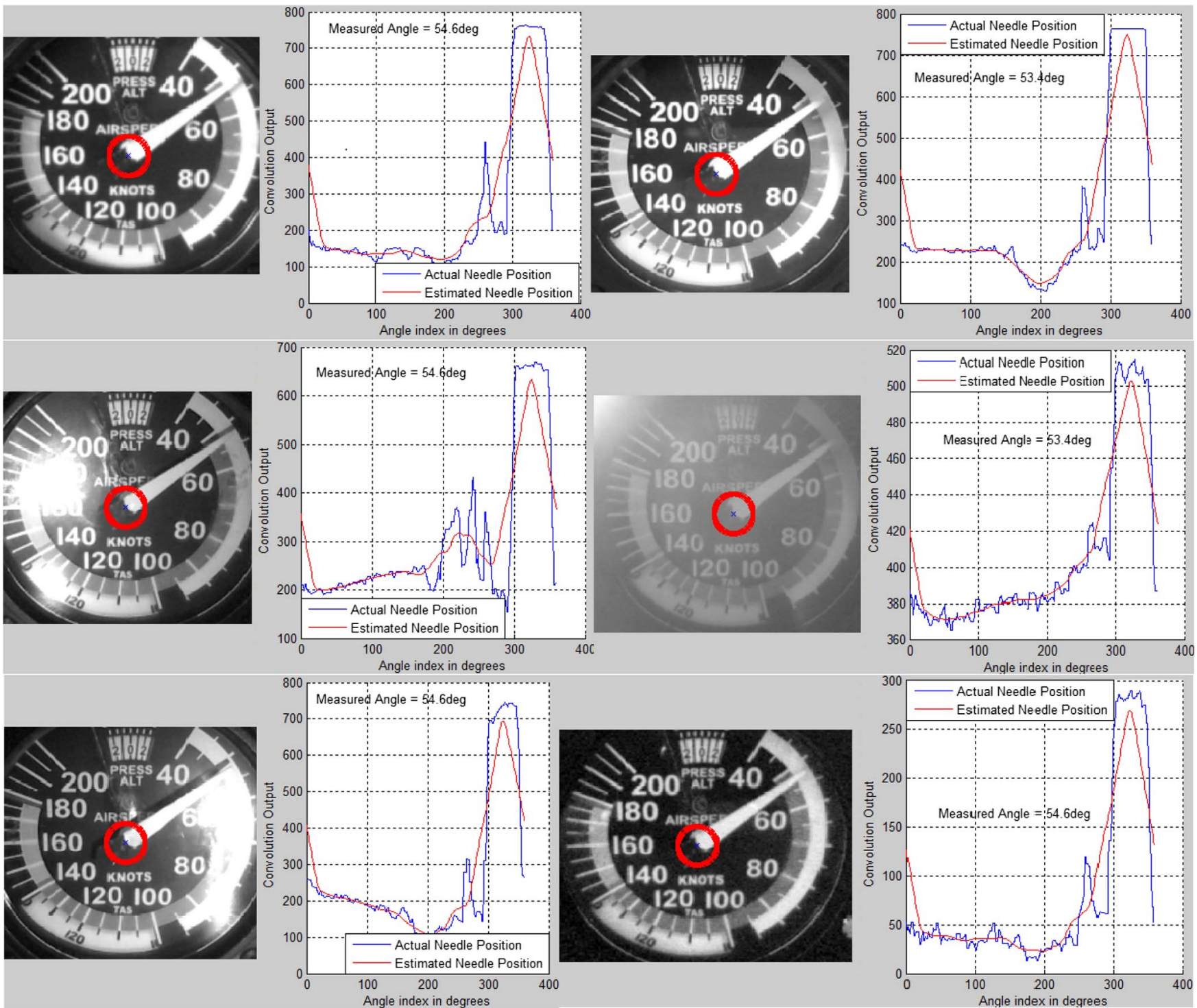

Fig. 9 Convolution based automated dial reading for varying light intensities and existence of specular highlights. 
according to the pilot instructions. In addition, the threshold value for the decision boundary for an instance to be considered as normal or hazard is defined using the receiver of characteristics (ROC) curve analysis as shown in Fig. 7.

The threshold value is varied from 0 to 100 with a lag of one unit, and the DSS performance is evaluated in terms of true positive rate (TPR) and false positive rate (FPR). An ROC curve for the decision boundary selection is achieved, which indicated the optimal compromise between TPR and FPR for the overall flight at 0.72 (i.e., $72 \%$ ) decision threshold value. Except the takeoff flight phase, where the decision threshold slightly decreases (i.e., 70\%), it remains almost stable for the rest of flight phases. This is because, in the takeoff phase, an additional turbulence is generated in the aircraft, resulting in the small vibration in the fixed-position camera devices. Statistical results in Table 2 demonstrate the impacts of different ADR approaches on the performance of the FG system. It can be observed that the best performance in terms of TPR, TNR, and accuracy is obtained using the convolution-based ADR that was introduced in our previous work [22]. Because of the rapid state variations in real flight, the computation time is also an important factor to be considered. Table 2 indicates that the optimal computation time of $0.41 \mathrm{~s}$ is achieved for the background-subtraction-based ADR approach [19]; however, it sacrifices the main aim by producing a 59.25\% accuracy rate. Comparatively, the convolution-based ADR produces the best accuracy rate of 96 and $100 \%$ in real flight and lab environments, respectively, along with an efficient computation time (i.e., $0.5 \mathrm{~s}$ ).

Existing cockpit warning indicators give indications in hazardous situations but need a considerable amount of attention from the pilot. In addition, it takes a lot of time to recognize the nature of problem and choose the right procedure [15,28], which consequently influences the flight safety in emergency situations. The proposed FG system resolves these issues by providing robust hazard detection in emergency situations, which reduces the pilot workload in terms of recognizing the nature of problem. For instance, providing direct information related to hazard type, its cause, and instructions to mitigate the abnormal situation will significantly reduce the time spent by the pilot to identify the hazard cause and eye scanning for subtasks. In comparison to the lab environment, the system generated $4 \%$ extra false alarms in real flight, hence reducing the system accuracy by $2.8 \%$. This reduction occurs due to the dynamics associated with the real flight environment such as light reflections and vibrations in the cameras due to air turbulence producing blurry images. Consequently, identification of the needle/gauge (i.e., ADR) becomes

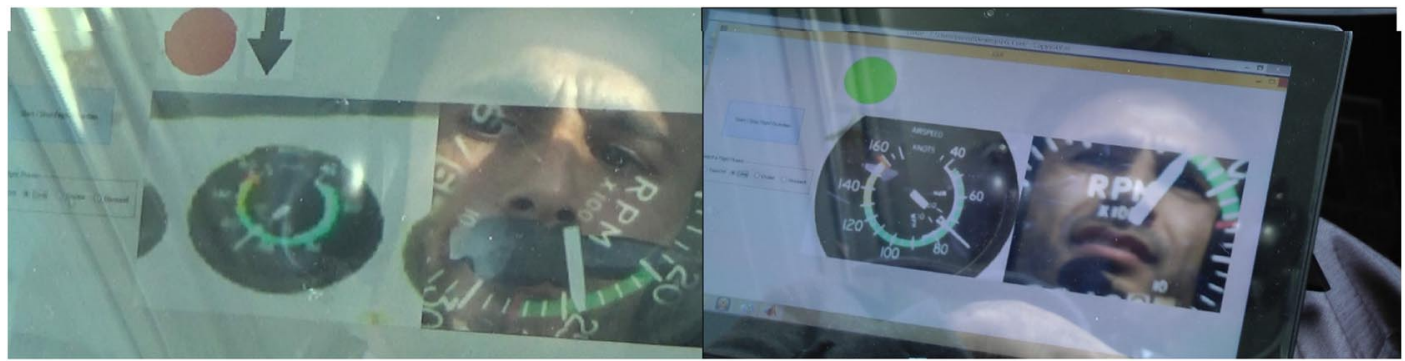

a) Climb normal airspeed

b) Climb high Airspeed

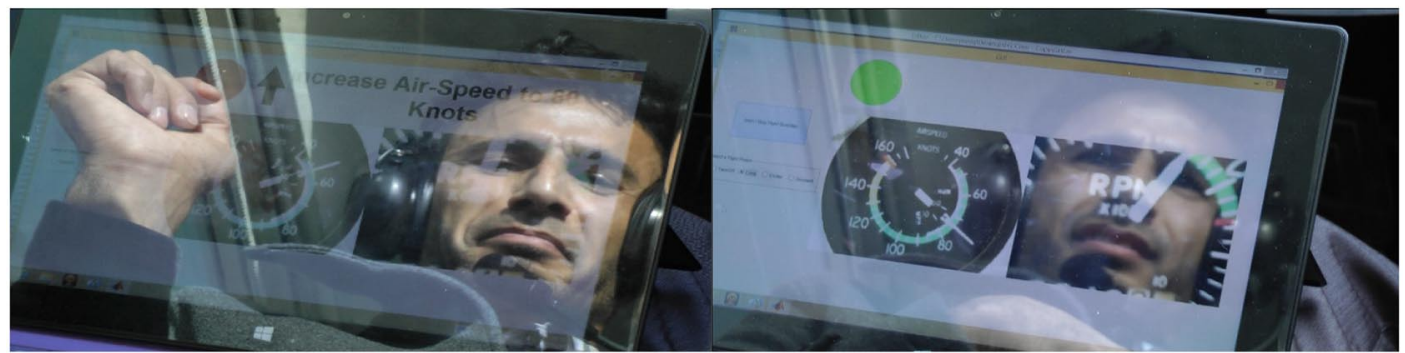

c) Cruise low airspeed

d) Climb normal RPM

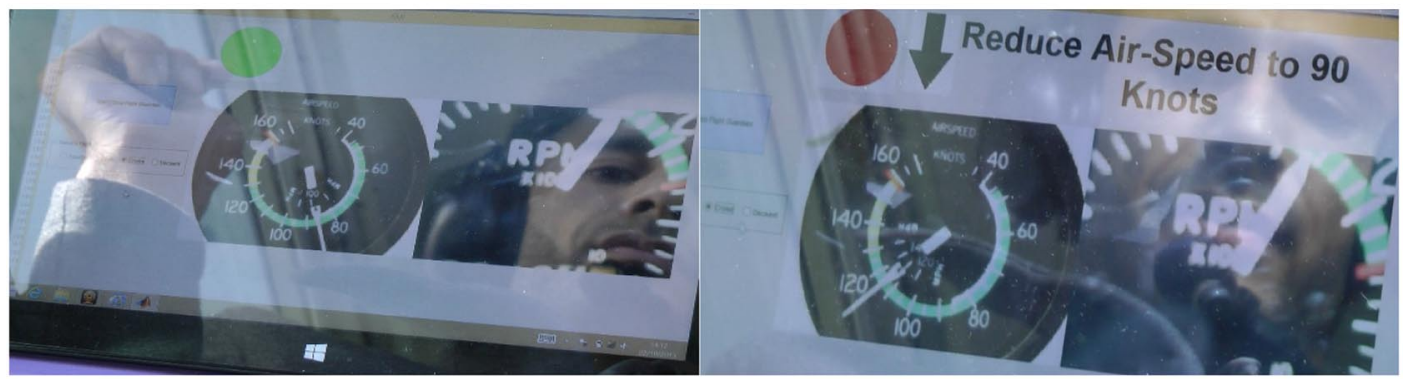

e) Cruise normal airspeed \& RPM

f) Cruise high airspeed

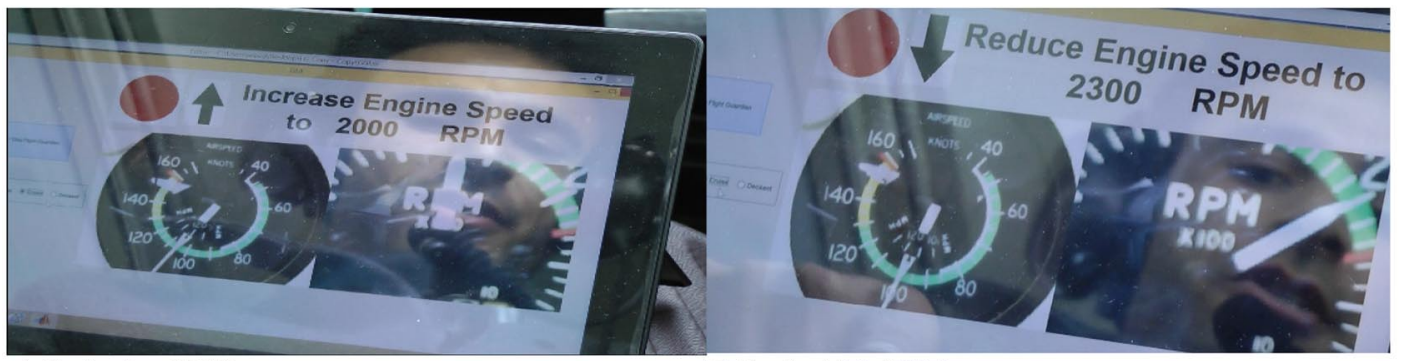

g) Cruise low RPM

h) Cruise high RPM

Fig. 10 Test cases for real flight evaluation of FG prototype in various hazardous situations in different flight phases. 
more challenging, resulting in 3.9\% false alarms. Despite these challenges in real flight, the $100 \%$ rate of hazardous situation detection proves the FG system significance in terms of flight safety improvements and pilot assistance as discussed in [11,12].

In addition to the aforementioned statistical results, the dial reading functionality is tested in the presence of specular highlights and varying light intensity while acquiring a uniform data stream using a flight simulator (FSX). A comparison between the uniform simulated data stream for ASp measurements (i.e., ground truth) acquired from FSX and predicted measurements by the convolution-based ADR system in the lab environment is presented in Fig. $\underline{8}$. It can be analyzed that the proposed ADR approach performed robustly in terms of precise overlapping of the ground-truth stream that produces a negligible rms error $(1.63 \mathrm{kt})$. Because the needle color in ASp and RPM instruments is white, it is challenging to differentiate the light reflections from the actual needle, as discussed by Vega et al. [20] and Gellaboina et al. [21]. However, the proposed convolution-based ADR along with the circular constraints makes it more accurate by minimizing the probability of the existence of light reflections in such a tiny area of the cockpit.

Statistical results achieved for varying lighting conditions in a lab simulated environment are presented in Table 3 . The intensity threshold for output images is adjusted by varying the contrast limits between 0 and 1 for two parameters $(\alpha, \boldsymbol{\beta})$. The first parameter increases the brightness level from the default (0) to a completely bright image by increasing its values, whereas the second parameter reduces the brightness level from the default (1) to a completely dark image by decreasing its values. The $p$-values are calculated using the t-test for the ground truth (GT) stream and ADR measurements while varying the $\alpha$ and $\beta$ parameters in the simulated environment. It can be observed that the convolution-based ADR produced consistency in output $p$-value with 0.01 (i.e., $1 \%$ significance level) in dynamic lighting conditions that indicated the strong confidence in support of the hypothesis that there is no significance difference between the GT and ADR measurements data streams. A slightly higher $p$-value (i.e., 0.02) is observed in the presence of direct specular light within the convolutional circular area around the needle that caused the misidentification of the needle. On the other hand, $p$-value is significantly increased when calculated for pattern matching $(p=0.19)$ and background subtraction $(p=0.49)$ based ADR approaches that indicate the significance difference between the GT data streams and the ADR measurements for these approaches. Likewise the root-mean-square error (RMSE) and false alarm rate are negligible for the convolution-based

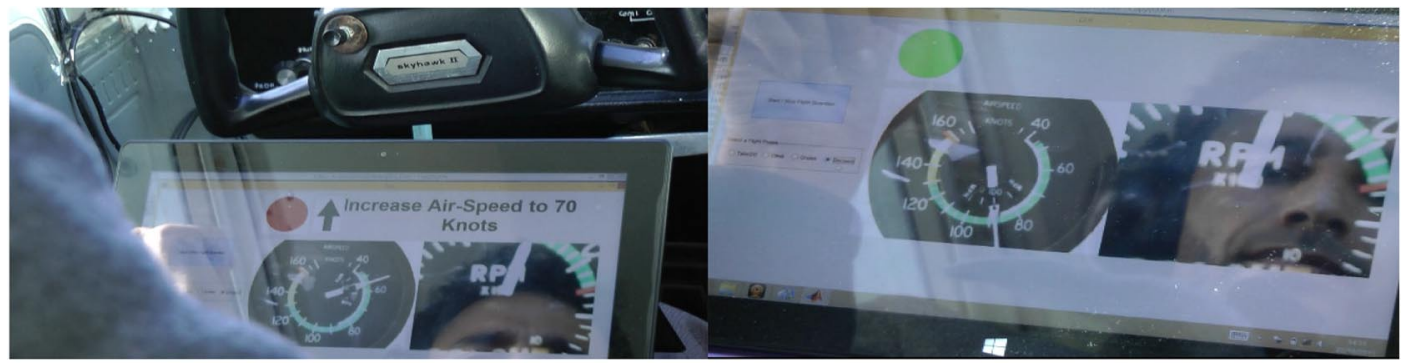

i) Descent low airspeed

j) Descent normal airspeed \& RPM

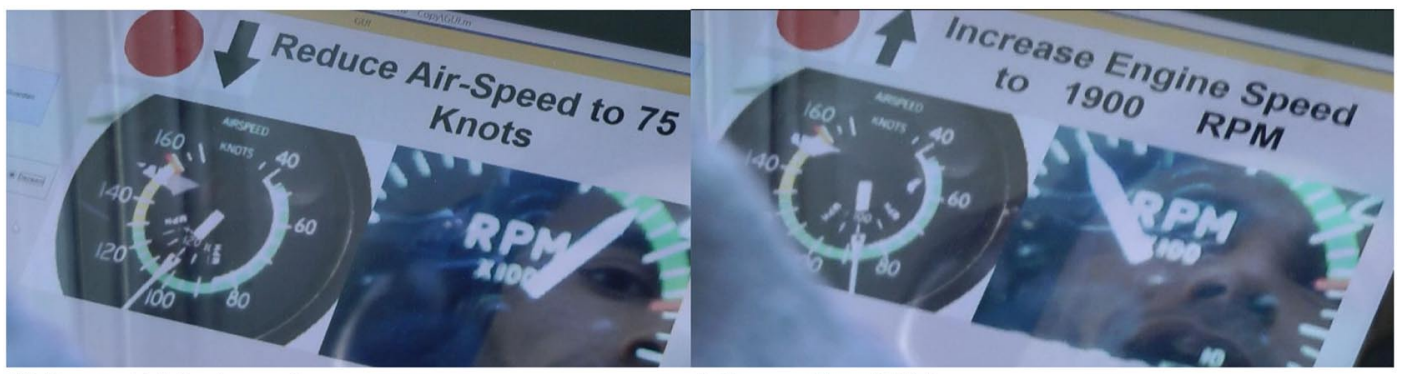

k) Descent high airspeed

I) Descent low RPM

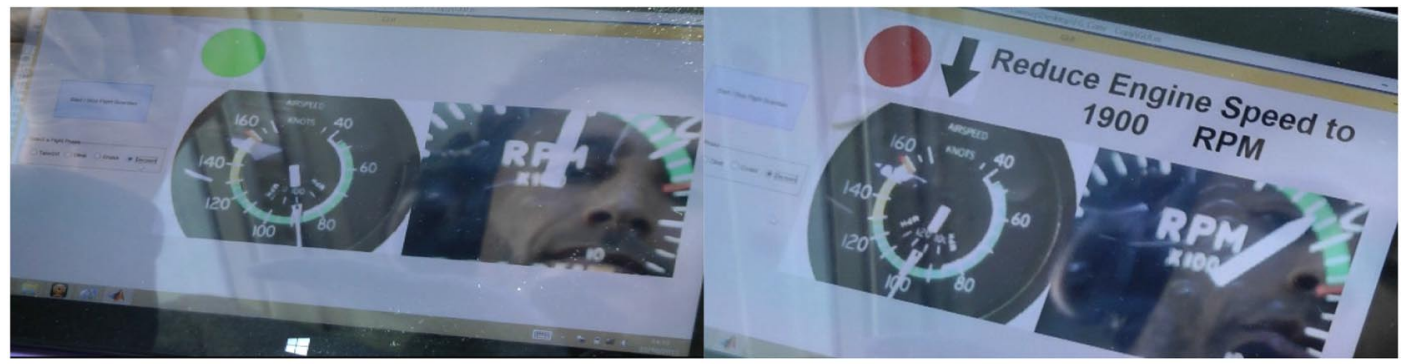

m) Descent normal RPM

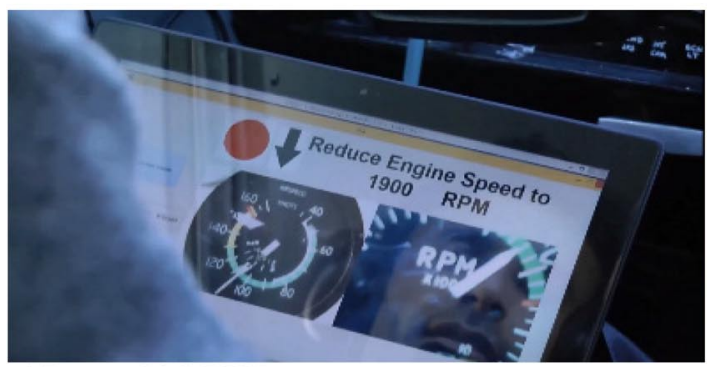

o) Descent high RPM n) Descent high RPM

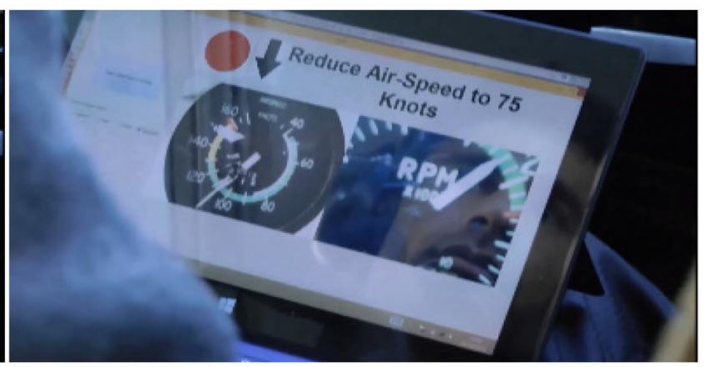

p) Descent high airspeed

Fig. 10 Continued. 
method that is not the case for other approaches. The hypothetical test and statistical measures are the evidence of the proposed convolution-based ADR approach over the existing background-subtraction and pattern-matching-based approaches.

Figure 9 shows multiple scenarios for the FG performance in the presence of specular highlights in the cockpit instruments and varying lighting conditions in the simulated flight environment. It is analyzed that the proposed ADR performed robustly in dynamic light intensity conditions by differentiating the pixels occupied by specular highlights and target object (needle) efficiently that consequently increases the performance of correct hazard identification and situation assessment.

In addition to the previously described statistical analysis of the results, multiple hazardous scenarios are created in a real flight environment using ASp and RPM instruments control to evaluate the FG system responses in emergency situations. Figure 10 demonstrates the visual snapshots taken for different hazardous situations created by the pilot during real flight while exceeding and dropping down the ASp and RPM values in climb, cruise, and descent flight phases. Despite the real flight dynamics (e.g., air turbulence and sunlight reflections), the proposed methodology produced a robust hazard diagnosis and provided corresponding visual and spoken instructions to the pilot to mitigate the abnormal situation.

Finally, a summary of the real flight test produced by both pilots stated that "During the live flight test of Flight Guardian the benefits to pilots of all abilities became clear. It is like having a second pilot issue timely observations and warnings about potentially hazardous situations - it has real potential to reduce pilot error in the cockpit and ultimately save lives". The pilot's report and the aforementioned detailed statistical results prove the proposed system efficiency and significance in terms of flight safety improvements and pilot assistance. These outcomes also serve as an enabler for the extension of this research in terms of its applicability to the variety of aircraft, fighting jets, commercial aircraft, and helicopters. Despite the future open-ended research interests, the collection of expert knowledge for each application domain, multiple flight phases, and additional cockpit instruments would be a challenging task that is needed to be considered in relation to FG system applicability to further domains.

\section{Conclusions}

The hypothesis underlying the principle objective as motivated for this research project was based on the development a regular monitoring platform for an aircraft that provides robust flight-deck awareness. The proposed system is able to intelligently generate voice-activated warnings and visual indications in hazardous situations. An experimental setup was built up comprising data acquisition, automated dial reading using video analysis, expert knowledge representation, belief combination, and decision making. Detailed statistical results were achieved that validated the proposed methodology in terms of flight safety improvements and its significance for the pilot assistance. For the first time, domain knowledge is presented for the cockpit instrument ranges with respect to different flight phases that might be of great interest for the related research. In spite of the robust outputs, there are some limitations associated with the selected methodology. For instance, the ADR task works well only for fixed-position camera devices focusing at the center position of the cockpit instrument. Similarly, the proposed system uses expert knowledge for two cockpit instruments for specific aircraft (i.e., Cessna C1772SP) that may be extended for the additional cockpit instruments and a variety of aircraft including commercial aircraft, helicopters, and fighting jets. These outcomes also serve as an enabler for future research on different aspects. A generic dial-reading approach may be developed that would automatically identify the desired cockpit instrument and rotate its image with respect to a fixed reference point (e.g., deep learning approaches). Likewise, the object detection can be further improved in a way that it handles the light reflections and vibrations more efficiently. In addition, the domain knowledge (i.e., data set for instrument ranges) might be extended to include more cockpit instruments to make the proposed system more generic in terms of automated flight phase detection and applicability to a wider range of civil and military aircraft.

\section{Acknowledgments}

Special thanks to the Great Circle Ltd., who were the industrial partner collaborating with the University of Central Lancashire on the development of FG. The FG project was funded (grant NWC2-S006) by the National Aerospace Technology Exploitation Programme (NATEP). NATEP is managed by the United Kingdom's national aerospace body ADS, supported by the U.K. regional aerospace alliances: FAC, NWAA, MAA, and WEAF.

\section{References}

[1] Wicken, C., Santamaria, A., and Sebok, A., "A Computational Model of Task Overload Management and Task Switching," Proceedings of the Human Factors and Ergonomics Society Annual Meeting, Vol. 57, No. 1, 2013, pp. 763-767. doi: $10.1177 / 1541931213571167$

[2] “Understanding the Distracted Brain: Why Driving While Using Hands-Free Cell Phones is Risky Behaviour," 2012, National Safety Council, http://www.nsc .org/safety_road/Distracted_Driving/Documents/Cognitive\%20Distraction\%20White\%20Paper.pdf [retrieved Aug. 2015].

[3] Loukopoulos, L., Dismukes, K., and Barshi, E., The Multitasking Myth: Handling Complexity in Real-World Operations, Ashgate, Surrey, England, U.K., 2015, pp. 13-14.

[4] Nikolic, M., Orr, J., and Sarter, N., "Why Pilots Miss the Green Box: How Display Context Undermines Attention Capture," International Journal of Aviation Psychology, Vol. 14, No. 1, 2004, pp. 39-52. doi:10.1207/s15327108ijap1401_3

[5] Miller, G. A., "The Magical Number Seven, Plus or Minus Two Some Limits on Our Capacity for Processing Information," Psychological Review by the American Psychological Association, Vol. 63, No. 2, 1956, pp. 81-97. doi: $10.1037 / \mathrm{h} 0043158$

[6] Sumwalt, R., Cross, D., and Lessard, D., "Examining How Breakdowns in Pilot Monitoring of the Aircraft Flight Path," International Journal of Aviation, Aeronautics, and Aerospace, ERAU Scholarly Commons, Vol. 2, No. 3, 2015, pp. 1-25. doi:10.15394/ijaaa.2015.1063

[7] "Safety Study: A Review of Flight Crew-Involved, Major Accidents of U.S. Air Carriers, 1978 Through 1990," National Transportation Safety Board, Rept. NTSB/SS-94/01, Washington, D.C., 1994, http://libraryonline.erau.edu/online-full-text/ntsb/safety [retrieved June 2015].

[8] Iwadare, K., and Oyama, T., "Statistical Data Analyses on Aircraft Accidents in Japan: Occurrences, Causes and Countermeasures," American Journal of Operations Research, Vol. 5, No. 3, 2015, pp. 222-245. doi:10.4236/ajor.2015.53018

[9] Boone, P., "Pilot Errors from a Pilot Perspective," June 2016, http://www.b737mrg.net/downloads/fly_the_dog.pdf [retrieved Aug. 2016].

[10] Oster, C. V., Strong, J. S., and Zorn, C. K., "Analysing Aviation Safety: Problems, Challenges, Opportunities," Research in Transportation Economics, Vol. 43, No. 1, 2013, pp. 148-164. doi:10.1016/j.retrec.2012.12.001 
[11] Grady, M., “NTSB Issues Glass Cockpit Safety Recommendations,” 2010, http://www.avweb.com/avwebflash/news/NTSBIssuesGlassCockpit SafetyRecommendations_202250-1.html [retrieved Sept. 2015].

[12] Sorrill, S. L., "What Safety Initiatives Have Been Introduced in Response to Glass Cockpits in General Aviation?" Southern Illinois Univ., Carbondale, IL, Paper 316, 2012, http://opensiuc.lib.siu.edu/gs_rp/316 [retrieved

[13] “Introduction of Glass Cockpit Avionics into Light Aircraft," National Transportation and Safety Board, Washington, D.C., 2012, http://www.ntsb.gov/safety/ safetystudies/SS1001.html [retrieved ].

[14] Kuchar, K. J., "White Paper on Multiple Independent Alerting Systems," 1998, http://www.mit.edu/ jkkuchar/white/whitepaper.html [retrieved ].

[15] Mulder, M., Pedroso, P., Mulder, M., and Passen, M. M. V., "Integrating Aircraft Warning Systems. Delft University of Technology, Faculty of Aerospace Engineering," 2000, pp. 179-183, https://scholar.google.com/citations?user=1nvtktYAAAAJ\&hl=nl [retrieved ].

[16] Abeloos, A. L. M., Mulder, M., and Paassen, M. M. V., "The Applicability of an Adaptive Human-Machine Interface in the Cockpit," Proceedings of the 19th European Annual Conf. on Human Decision Making and Manual Control, 2000, pp. 193-198.

[17] Thatcher, S., "An Artificial Intelligent Paradigm for Systems Safety in the Cockpit,” AERO Lab, Univ. of South Australia, 2008, http://citeseerx.ist.psu.edu/ viewdoc/download?doi=10.1.1.102.7992\&rep=rep1\&type=pdf [retrieved

[18] Marie, S. L., "An Investigation of the Effectiveness of a Strobe Light as an Imminent Rear Warning Signal," M.S. Thesis, Virginia Polytechnic Inst. and State Univ., Blacksburg, VA, 2000, http://scholar.lib.vt.edu/theses/available/etd-12012000-084911/unrestricted/Literature.pdf [retrieved March 2015].

[19] Feng, H., and Zhao, J., "Application Research of Computer Vision in the Auto-Calibration of Dial Gauges," International Conf. on Computer Science and Software Engineering, Vol. 2, 2008, pp. 845-848. doi:10.1109/CSSE.2008.350

[20] Vega, R. O., Ante, G. S., Morales, L. E. F., and Sossa, H., "Automatic Reading of Electro-Mechanical Utility Meters," Proceedings of the 12th Mexican International Conf. on Artificial Intelligence, 2013, pp. 164-170. doi:10.1109/MICAI.2013.28

[21] Gellaboina, M. K., Swaminathan, G., and Venkoparao, V., "Analogue Dial Gauge Reader for Handheld Devices," Proceedings of the IEEE 8th Conf. on Industrial Electronics and Applications, IEEE Publ., Piscataway, NJ, 2013, doi:10.1109/ICIEA.2013.6566539

[22] Khan, W., Ansell, D., Kuru, K., and Amina, M., "Automated Aircraft Instrument Reading Using Real Time Video Analysis," Proceedings of the IEEE Conf. on Intelligent Systems, IEEE Publ., Piscataway, NJ, Sept. 2016, doi:10.1109/IS.2016.7737454

[23] “Aircraft Information Booklet, Cessna 172S," 2013, http://www.airborne-aviation.com.au/resources/aircraft/booklet_cpq.pdf [retrieved Dec. 2014].

[24] "Pilot's Operating Handbook, Cessna 172N," Skyhawk, 1978, http://www.skywarriorinc.com/downloads/POH\%20BOOKS/172N\%20POH.pdf [retrieved Feb. 2015].

[25] Mandal, S. N., Choudhury, J. P., and Chaudhuri, S. R., "In Search of Suitable Fuzzy Membership Function in Prediction of Time Series Data," International Journal of Computer Science Issues, Vol. 9, No. 3, 2012, pp. 293-302.

[26] Foley, B. G., "A Dempster-Shafer Method for Multi-Sensor Fusion,” M.Sc. Thesis, Dept. of Mathematics and Statistics, U.S. Air Force Inst. of Technology, Wright-Patterson AFB, OH, 2012, http://www.dtic.mil/dtic/tr/fulltext/u2/a557749.pdf [retrieved April 2015].

[27] Chen, Q., Whitbrook, A., Aickelin, U., and Roadknight, C., "Data Classification Using the Dempster-Shafer Method," Journal of Experimental \& Theoretical Artificial Intelligence, Vol. 26, No. 4, 2014, pp. 493-517. doi:10.1080/0952813X.2014.886301

[28] Dismukes, K., Young, G., and Sumwalt, R., “Cockpit Interruptions and Distractions,” ASRS Directline, No. 10, 1998, pp. 4-9, https://asrs.arc.nasa.gov/docs/ dl/DL10.pdf [retrieved ].

J. P. How

Associate Editor 


\section{Queries}

1. AU: The title cannot begin with an article (e.g., "the").

2. AU: Please check that the copyright (C) information is correct.

3. AU: Please check that the authors' affiliations and footnotes are correct.

4. AU: Acronyms are not allowed in the Abstract.

5. AU: The URL provided in this footnote does not work. Please provide a valid URL and date of retrieval.

6. AU: Acronyms that are defined and used only once (e.g., FAA) are removed.

7. AU: Please note that subsections are not allowed in the Introduction and have been removed.

8. AU: Please check the status of your request for figure coloration (in print, online only, both, or neither) and confirm that the figures in your proof are correct.

9. AU: If the figures in your paper are to appear in black $\&$ white or in color online only (i.e., not color in print), please remove from the main text and all figure captions any references to color in the figures (e.g., "red circle"). If figures are to appear in color in print, then these references to color in the figures can remain.

10. AU: Please provide the date of retrieval for the URL in this footnote.

11. AU: The captions to Tables 1-3 exceed the maximum length of 12 words. Please reduce them. You may consider moving some information to the main text.

12. AU: Vertical spanning of cells in tables is not allowed.

13. AU: All figures must be cited in numerical order in the main text. Figure 4 was not cited. Please cite this figure in numerical order in the main text.

14. AU: Please spell out "TP", "TN", "FP", and "FN" in Table 2, or provide the definitions in the text.

15. AU: The layout of Table 3 was modified slightly to better adhere to AIAA style. Please confirm that your intended meaning is retained.

16. AU: The final section must be called "Conclusions".

17. AU: Please spell out "ADS", "FAC", "NWAA", "MAA", and "WEAF".

18. AU: Please check if Ref. [1] is a journal or a conference proceedings. If it is a journal, it is acceptable as is. If it is a conference proceedings, please remove issue number, and provide name and location of publisher (not conference host).

19. AU: Please provide the location of the publisher for Refs. [2, 24].

20. AU: The URL provided for Ref. [7] does not work. Please provide a valid URL and date of retrieval for this reference. 
21. AU: Please provide the name and location of the publisher for Refs. [9, 11, 23].

22. AU: Please provide the date of retrieval for the URLs in Refs. [12, 12, 28].

23. AU: Please provide the name and location of the publisher as well as the date of retrieval for the URL in Ref. [14].

24. AU: The URL provided for Ref. [15] does not lead to the cited document. Please update the URL accordingly and provide the date of retrieval, as well as the name and location of the publisher.

25. AU: If Refs. [16, 19, 20] are published proceedings, please provide the name and location of the publisher (not the conference host) and the page numbers used. If they are conference papers, please provide the paper number and the organizer's name. If they are CD-ROMs, please provide the name and location of the CD-ROM producer.

26. AU: Please provide the location of the publisher and the date of retrieval for the URL in Ref. [17].

27. AU: Please provide the pages for Refs. [21, 22]. 